\title{
Erratum: Identity method for particle number fluctuations and correlations [Phys. Rev. C 84, 024902 (2011)]
}

\author{
M. I. Gorenstein
}

(Received 22 December 2017; published 14 February 2018)

DOI: 10.1103/PhysRevC.97.029903

There was a misleading notation in Eq. (10),

$$
u_{j i}^{s} \equiv \frac{1}{\left\langle N_{i}\right\rangle} \int d m w_{j}^{s}(m) \rho_{i}(m),
$$

with $s=1,2$. Index $s=2$ in this formula did not correspond to the square sign, and that implied $u_{j i}^{2} \neq\left(u_{j i}\right)^{2}$. It led to mistakes in several subsequent equations. This happens because the expressions of $\left(u_{j i}\right)^{2}$ were mixed up with those of $u_{j i}^{2}$. In order to correct everything, I suggest the following modifications. Equation (10) should be replaced by

$$
u_{j i} \equiv \frac{1}{\left\langle N_{i}\right\rangle} \int d m w_{j}(m) \rho_{i}(m), \quad \overline{u_{j i}^{2}} \equiv \frac{1}{\left\langle N_{i}\right\rangle} \int d m w_{j}^{2}(m) \rho_{i}(m),
$$

and the first equation in Eq. (12),

$$
\left\langle W_{j}^{2}\right\rangle-\sum_{i=1}^{k}\left\langle N_{i}\right\rangle\left[u_{j i}^{2}-\left(u_{j i}\right)^{2}\right] \equiv b_{j},
$$

with $u_{j i}^{2} \neq\left(u_{j i}\right)^{2}$ in Eq. (12), should be replaced by

$$
\left\langle W_{j}^{2}\right\rangle-\sum_{i=1}^{k}\left\langle N_{i}\right\rangle\left[\overline{u_{j i}^{2}}-u_{j i}^{2}\right] \equiv b_{j}
$$

After these replacements, one has $u_{j i}^{2}=\left(u_{j i}\right)^{2}$ in Eq. $\left(12^{*}\right)$ and in all subsequent equations of the original paper. The expressions $\overline{u_{j i}^{2}}$ are only entered in $b_{j}$ quantities, and with new Eqs. $\left(10^{*}\right)$ and $\left(12^{*}\right)$, all subsequent formulas become correct. Note that the corrections in formulas (10) and (12) proposed in this Erratum make all equations self-consistent. These corrections do not change, however, any statements and/or conclusions made in the original paper.

I am thankful to A. Ohlson and A. Rustamov for pointing out the problem. 